\section{Pressure sores}

\section{Not considered a priority by medical staff}

EDrToR,-R K Vohra and C N McCollum's review of pressure sores is a timely reminder of the continuing silent epidemic of pressure sores both in hospital and in the community. ${ }^{1}$ Pressure sores impose two main penalties, financial and human. The financial costs are high and result from additional treatment, a prolonged stay in hospital, and a further demand on clinic (nursing) time. Prevention of pressure sores is based on constant vigilance and early action, with awareness being the keynote of success.

A colleague and I conducted a detailed survey with a semistructured questionnaire in a university teaching hospital; medical staff responded poorly to the questionnaire because pressure sores were not considered to be a priority. ${ }^{2}$ Only about a quarter of medical staff routinely assessed patients with regard to their risk of pressure sores, though most nursing and therapy staff performed routine assessments. Hospital staff other than nursing staff were unaware of any protocols for assessing pressure sores. Most of the respondents thought that the cost of treating an inpatient with a pressure sore was less than $£ 1000$; this indicates their unawareness of the true cost.

It is prudent to apply low technology early rather than high technology at a later stage. If equipment is required it should be compatible with existing equipment, and unless costs are realistic the strategy is doomed to failure.

2 Kulkarni J, Philbin M. Pressure sore awareness survey in a university teaching hospital. foumal of Tissue Viability 1993;3: 77-9.

\section{Knowledge of risk factors may not improve management}

EDITOR,-Greater understanding of both " the pathophysiology and the risk factors associated with pressure necrosis has led to the development of various scoring systems aimed at identifying patients at high risk. In their review of pressure sores $\mathrm{R} \mathrm{K}$ Vohra and C N McCollum postulate that the widespread introduction of these risk scales into clinical practice may lead to increased awareness of the problem and facilitate more effective preventive care. ${ }^{1}$

Our experience, however, is that the introduction of risk scales has not invariably led to the use of appropriate preventive measures. A recent study from this department compared patients' Waterlow scores with the pressure relieving care given $\mathrm{V}$ Banks et al, proceedings of the fourth European Wound Management Association, Copenhagen, 1994). Little correlation was found between the risk that had been assessed and the prophylactic measures used. Use of pressure relieving equipment was based more on availability than on need. Less than half of patients studied in an acute unit were receiving appropriate treatment despite scores indicating a high risk. Measures provided for patients in the community were found

We prefer short letters that relate to a recently published article and we are unlikely to publish letters longer than 400 words and containing over five references. Letters may be shortened. Your letters should be typed with double spacing and include a word count. All authors need to sign the letter and provide one current appointment and address. We encourage you to declare any conflict of interest. Please enclose a stamped addressed envelope if you require an acknowledgment.

to be almost universally inadequate for all categories of risk.

We contend that the continued high incidence of pressure sores is as much related to variable distribution of equipment as to lack of knowledge regarding risk factors.

$$
\begin{array}{r}
\text { JUDITH NASH } \\
\text { Research fellow } \\
\text { PHIIIP BARAGWANATH } \\
\text { Research fellow } \\
\text { ROBERT A SALAMAN } \\
\text { Research fellow }
\end{array}
$$

Wound Healing Research Unit,

Department of Surgery,

University of Wales College of Medicine, Cardiff CF4 4XN

1 Vohra RK, McCollum CN. Pressure sores. BMf 1994;309: 853-7. (1 October.)

\section{Alternating pressure systems are useful}

EDrToR, - In their article on pressure sores $\mathrm{R} \mathrm{K}$ Vohra and $\mathbf{C ~ N}$ McCollum state that alternating systems should be avoided. ${ }^{1}$ The article on which the authors base their comments related to pressure relieving equipment not used in Britain: the particular alternating system cell was only $3.8 \mathrm{~cm}$ thick ${ }^{2}$ and is a mattress overlay system.

Recent studies comparing alternating pressure overlays showed that all tested, except the small cell ripple, were acceptable to patients and staff and suitable for patients at medium risk of developing pressure damage. ${ }^{3}$ I suggest that the alternating system in question should be used only for patients at medium risk.

Unfortunately, Britain does not have a national code of practice relating to the purchase of pressure relieving equipment, and purchasers have little information on which to base decisions. In many hospitals in Britain, however, alternating mattress replacement systems are used to prevent pressure damage for patients at high risk, and two systems (the Pegasus and Nimbus) have been proved to give pressure readings below $32 \mathrm{~mm} \mathrm{Hg}$ at some time in the cycle. ${ }^{3}$ An extensive study carried out in Germany to test the effectiveness of specialised bed systems measured interface pressures and transcutaneous partial pressures of oxygen plus carbon dioxide. The results clearly showed that the Pegasus Airwave alternating mattress replacement system is capable of temporarily generating pressures in the region of zero, so that hyperaemia of the previously underperfused area is possible. Clinical data also show that the Nimbus alternating mattress replacement system is suitable for patients at high risk of developing pressure damage. $^{3}$

In this NHS trust in the past 18 months we have achieved a reduction in the overall prevalence of pressure damage. The vital key elements to success are education of staff and patients and use of a combination of support surfaces, depending on assessment of each patient.

ELAINE BETHELI

City Hospital NHS Trust

Tissue viability clinical nurse specialist

Birmingham B18 7QH

1 Vohra RK, McCollum CN. Pressure sores. BMF 1994;309: 853-7. (1 October.)

2 Sioleronko S, Quinn A, Burns K, Frances RD. Effects of position and mattress overlay on sacral and heel pressures in a clinical and mattress overlay on sacral and heel pressur

population. Res Nurs Health 1992;15:245-51.
3 Swain I, Nash R, Robertson J. Assessment of support surfaces: Swain I, Nash R, Robertson J. Assessment of support surfaces:
comparison of Nimbus and Pegasus (Airwave) mattress. comparison of Nimbus and Pegasus

4 Schregel $W$, Hube $M$, Finstervalder $H$. Static and dynamic systems for ITU care patients. Foumal of Tissue Viability 1993;4:106-14.

\section{Compare maximum and minimum pressures}

EDrToR,- - R K Vohra and C N McCollum's use of measurements of contact (interface) pressure to express the effectiveness of pressure redistributing devices in preventing pressure sores have repeated common misconceptions of the use of sùch data. ${ }^{1}$ The authors comment that the use of alternating air mattresses should be avoided because of the high mean contact pressures generated compared with those generated by static devices such as water mattresses. This recommendation is based on flawed evidence. Crow and I reported that alternating and static devices should not be compared on the basis of mean contact pressures because such measurements are inadequate to represent the different principles of operation of the two classes of pressure redistributing device. ${ }^{2}$ If devices are to be compared on the basis of contact pressure both the maximum and minimum applied pressures must be compared; of these, the minimum applied pressure may be most important.' Even when appropriate measures are used to compare products it is unwise to place much emphasis on measurements of contact pressure, given the well documented problems in making accurate measurements and the unproved validity of such measures as expressions of the effectiveness of equipment.

As with other aspects of medical technology, the evaluation of pressure redistributing devices must rest on evidence of outcomes in well designed randomised controlled trials and not spurious intermediate measures such as the contact pressure between patients and their beds.

Pegasus Airwave,

Waterlooville,

Hampshire PO7 7XX

1 Vohra RK, McCollum CN. Pressure sores. BMf 1994:309: 853-7. (1 October)

2 Crow RA, Clark M. Current management for the prevention of pressure sores. In: Boder DL, ed. Pressure sores: clinical practice and scientific approach. London: Macmillan, 1990:43-54.

3 Clark M, Rowland LB. Comparison of contact pressures measured at the sacrum of young and elderly subjects. f Biomed Eng 1989;11:197-9.

4 Clark M. Problems associated with the measurement of interface (or contact) pressure. foumal of Tissue Viability 1994;4:37-43.

^Pegasus Airwave manufactures alternating air mattresses. 